\title{
Tailoring the magnetoresistance of MnAs/GaAs:Mn granular hybrid nanostructures
}

\author{
C. Michel, ${ }^{1,2, a)}$ M. T. Elm, ${ }^{2}$ B. Goldlücke ${ }^{3}$ S. D. Baranovskii, ${ }^{1}$ P. Thomas, ${ }^{1}$ W. Heimbrodt, ${ }^{1}$ \\ and P. J. Klar $^{2}$ \\ ${ }^{1}$ Department of Physics and Material Sciences Center, Philipps-University, Renthof 5, \\ 35032 Marburg, Germany \\ ${ }_{2}^{2}$ Institute of Experimental Physics I, Heinrich-Buff-Ring 16, Justus-Liebig University, \\ 35392 Giessen, Germany \\ ${ }^{3}$ MPI for Computer Science, Stuhlsatzenhausweg 85, 66123 Saarbrücken, Germany
}

(Received 10 April 2008; accepted 7 May 2008; published online 6 June 2008)

\begin{abstract}
The magnetoresistance properties of GaAs:Mn/MnAs granular hybrid structures consisting of ferromagnetic MnAs clusters within a paramagnetic GaAs:Mn host differ considerably from those of paramagnetic and ferromagnetic $(\mathrm{Ga}, \mathrm{Mn})$ As alloys. We analyze the magnetoresistance effects on the basis of a resistor network model. Typical experimental findings are reproduced and their dependence on cluster density and random spatial arrangement of the clusters are revealed. Controlled spatial positioning of the MnAs clusters within the GaAs:Mn host offers interesting opportunities for optimizing the magnetoresistance properties for applications and for overcoming problems of miniaturization arising from cluster statistics. (C) 2008 American Institute of Physics. [DOI: $10.1063 / 1.2937128]$
\end{abstract}

Granular hybrid structures are currently of interest for devices as the material properties can be tuned in a wide range. ${ }^{1-6}$ The tunability arises from the larger number of degrees of freedom in a composite hybrid compared to a single-phase material. Additional degrees of freedom are the mean distance between clusters or the cluster size. The latter contribute to a large extent to the properties of the hybrid. ${ }^{7,8}$ For example, the planar magnetoresistance (MR) of granular thin films consisting of a diamagnetic matrix material, either insulating or metallic, with ferromagnetic inclusions is strongly related to the giant MR (GMR) effect of corresponding ferromagnetic/diamagnetic/ferromagnetic triple layer systems. ${ }^{9}$

So far, granular materials have been mainly used in macroscopic devices, i.e., where the mean distance between clusters is much smaller than the characteristic device size. Macroscopic devices allow one to take advantage of the tunability of the hybrid properties while avoiding problems due to the statistical fluctuations in cluster number, size, etc. Moore's law is a key issue of today's technology and progress and prosperity depend significantly on the miniaturization of devices according to the technology road maps. ${ }^{10}$ This somewhat suggests that conventionally synthesized granular hybrids will play no role in the long run. However, recent progress in nanostructure growth offers novel ways of controlling size and position of clusters in host materials on a submicrometer scale, e.g., selective-area metal-organic vapor-phase epitaxy (MOVPE). ${ }^{11,12}$

In our report, we discuss possible ways of tailoring the MR of paramagnetic-ferromagnetic GaAs:Mn/MnAs granular hybrids. Such hybrids with statistical arrangements of clusters can be obtained by conventional MOVPE or by molecular beam epitaxy followed by an annealing step. Selective-area MOVPE offers a possibility to almost overcome the statistical element. However, to optimize the hybrid

${ }^{\text {a)} E l e c t r o n i c ~ m a i l: ~ c h r i s t o p h . m i c h e l @ p h y s i k . u n i-m a r b u r g . d e . ~}$ properties for a specific application, it is essential to develop corresponding theoretical models. Such a model at hand, predictions of the MR properties can be made and additional insight into the interaction mechanisms between cluster and matrix can be obtained. The aim of this letter is to demonstrate this theoretical optimization procedure in the case of the MR of granular GaAs: Mn/MnAs hybrids.

We use the resistor-network model that has been successfully employed earlier for describing quantitatively the transport properties of $\mathrm{Ga}_{1-x} \mathrm{Mn}_{x} \mathrm{As}$ alloys and random granular MnAs/GaAs:Mn hybrids. ${ }^{13,14}$ In the case of hybrids, one needs to account for the properties of the semiconducting GaAs:Mn host, ${ }^{14}$ for those of the MnAs clusters, and for the interplay of cluster and the host material. The MnAs cluster is assumed to be half-metallic with constant majorityband conductivity and a minority band which shows activated transport with an activation energy larger than that of the matrix. Furthermore, we account for the influence of (i) the formation of a Schottky barrier at the cluster-host interface $^{15}$ which decays with increasing distance from the cluster surface and causes a trapping of free carriers, (ii) the interplay of a dipolar magnetic field of the ferromagnetic cluster with the magnetic moments of Mn ions in the surrounding paramagnetic host which leads to a local giant Zeeman splitting (GZS) of the host band states, ${ }^{15}$ and (iii) different spin orientations of cluster and host majority carriers. ${ }^{13,15}$ The model system is divided into cubic cells of equal size with either cluster or matrix properties. The Mn ions are distributed randomly over the matrix cells and the resistivity of each matrix cell is calculated as $\rho=(n e \mu)^{-1}$, where $n$ is the local carrier concentration, $e$ the elementary charge, and $\mu$ a band mobility. The total resistivity is calculated by applying Kirchhoff's knot law. The temperature dependence of the spin conservation of the free carriers in the transport process is accounted for by a weighted average of the total resistance in the case of perfect spin conservation (Mott's two-channel model), $\rho_{\mathrm{sc}}$, and spin relaxation on the length of a network cell, $\rho_{\mathrm{nc}}$. The weighing factor depends on 


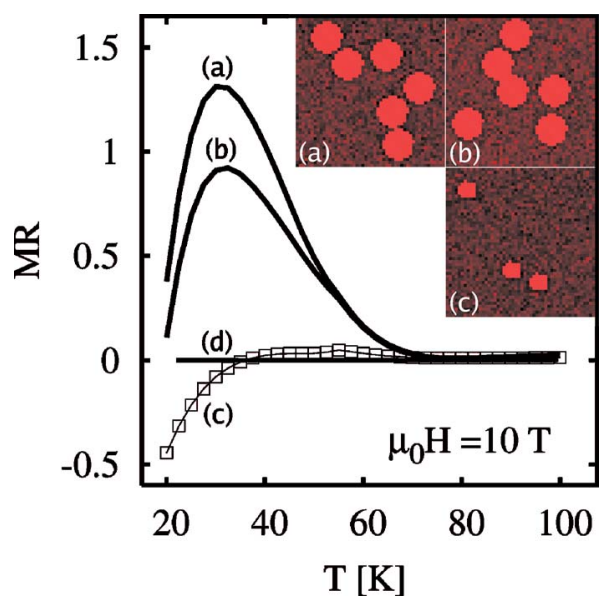

FIG. 1. (Color online) Calculated $\mathrm{MR}$ at $\mu_{0} H=10 \mathrm{~T}$ vs temperature for different cluster densities $x_{C}$ and random distributions of the MnAs clusters as depicted in the insets. [(a) and (b)] $x_{C}=22 \%$; (c) $x_{C}=3.5 \%$; (d) $\mathrm{Ga}_{0.994} \mathrm{Mn}_{0.006} \mathrm{As}$ without clusters.

the Boltzmann term $\exp \left(-\Delta E / k_{B} T\right)$, where $\Delta E$ is given by the average GZS of the valence band states of the GaAs:Mn matrix. For the results presented here, we use arrays of 50 $\times 50$ network cells. A detailed description of the model can be found in Ref. 13 .

The findings of MR experiments on GaAs: Mn/MnAs hybrids with randomly arranged MnAs clusters can be summarized as follows: ${ }^{16-19}$ (i) MR effects of hybrids are considerably larger than those of the pure host matrix; (ii) a strongly enhanced resistance of hybrid samples compared to the pure hosts is observed at low temperatures leading to a pronounced non-Arrhenius behavior at low temperatures; (iii) large negative MR effects occur at low temperatures in hybrids $(T<30 \mathrm{~K})$, whereas (iv) pronounced positive MR effects up to several hundred percent are present in the intermediate temperature regime $(30<T<100 \mathrm{~K})$ which decrease rapidly toward room temperature. Findings (ii) and (iii) can be qualitatively explained by trapping of holes at the cluster-matrix interface due to the formation of a Schottky barrier and an additional trapping due to the local GZS of the matrix states caused by the dipolar field of the cluster in its vicinity. ${ }^{13}$ This trapping is partly lifted by an external magnetic field due to the saturation of the GZS at larger fields. The carriers are released leading to a decrease of the resistivity with field, i.e., a negative MR effect. Finding (iv) arises from the difference in majority and minority spins of matrix and cluster material. In an applied magnetic field, the majority spins of the matrix cannot pass through the clusters. This effect decreases as the GZS in the matrix breaks down with increasing temperature. These experimental findings can be well reproduced by the model as is demonstrated in Fig. 1, where MR values at $10 \mathrm{~T}$ are plotted as a function of temperature between 20 and $100 \mathrm{~K}$. The curves are calculated for cluster densities $x_{C}$ of $22 \%$ and six spatially randomly positioned clusters [curves (a) and (b)] of $3.5 \%$ and three clusters [open symbols, curve (c)], and for a pure $\mathrm{Ga}_{0.994} \mathrm{Mn}_{0.006}$ As matrix without clusters [curve (d)].

Performing systematic calculations for various random cluster arrangements in dependence on cluster density $x_{C}$ and magnitude of the Schottky barrier and/or cluster field yields the following consistent scheme depicted in Fig. 2. The negative MR, which is caused by the magnetic-field and tem-

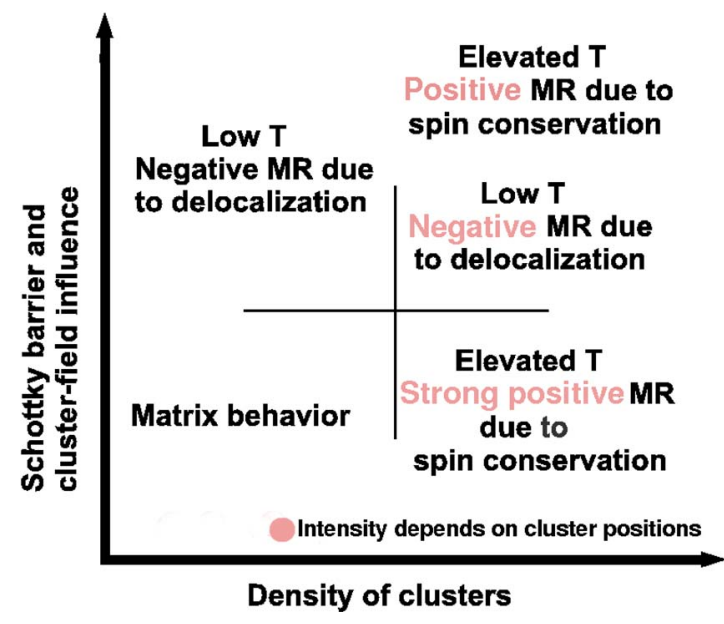

FIG. 2. (Color online) Illustration of the dominant MR effects of MnAs/GaAs:Mn hybrids in dependence on Schottky barrier and/or the magnetic field of the clusters and cluster density.

perature induced delocalization of trapped carriers, increases with the depth of the trapping potential. The positive MR becomes dominant at higher cluster densities when the areas of the matrix affected by the clusters start to overlap, i.e., a percolation path between trapping regions is established. At these higher cluster densities, current path blocking by the clusters due to the combination of spin conservation and the different spin-dependent densities of states of cluster and matrix becomes dominant. Thus, the positive MR can be considered a geometric effect, as the extraordinary MR (EMR) observed, e.g., in $n$-type InSb:Te. ${ }^{20}$ However, it should be noted here that due to the mobilities in GaAs:Mn and the corresponding small Hall angles, the mechanism behind the positive MR is different from that responsible for the EMR effect. The positive MR contribution vanishes at low cluster concentrations as the influence of the clusters on the main percolation path of the carriers through the system becomes negligible.

The dependence of the positive MR effect on geometry is also reflected by the difference of curves (a) and (b) in Fig. 1. This difference arises solely from the different spatial arrangements of clusters within the model system of $50 \times 50$ cells as all the material parameters are kept constant in the calculations. Fluctuations of the magnetoresistance effects caused by a random incorporation of $\mathrm{Mn}$ in the GaAs:Mn are of minor importance, i.e., an order of magnitude smaller than those due to a controlled spatial arrangement of the MnAs clusters shown in the figure. ${ }^{21}$ The insets, labeled (a) and (b), of the figure show the two arrangements corresponding to the curves, voltage drop in all insets from left to right. The dependence on the arrangement of the clusters within the model system is less pronounced at the lower cluster density of $x_{C}=3.5 \%$ where carrier trapping is the dominant effect caused by the clusters. This is easily understood as basically the areas of the matrix affected of the clusters do not overlap at this cluster density and the main current path through the sample is not affected by the clusters [see inset (c)]. The fluctuations of the MR observed in ten different runs of the calculations at this $x_{C}$ are equivalent to the size of the open symbols on the scale of the figure.

Typical sizes of the MnAs clusters in MOVPE grown GaAs:Mn/MnAs hybrids are of the order of $60 \mathrm{~nm}$ in diameter. Thus, the $50 \times 50$-model system would roughly 


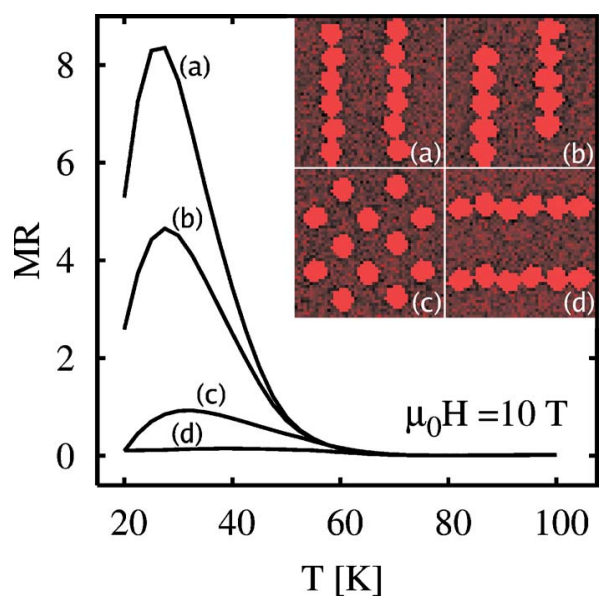

FIG. 3. (Color online) Calculated MR at $\mu_{0} H=10 \mathrm{~T}$ between 20 and $100 \mathrm{~K}$ for four different tailored cluster arrangements [(a)-(d)] shown as insets. Cluster density $x_{C}=22 \%$.

correspond to a sample size of about $0.3 \times 0.3 \mu \mathrm{m}^{2}$, which is typical for miniaturized devices. This means that, due to statistics of the spatial cluster arrangement, miniaturized devices based on such granular GaAs: Mn/MnAs hybrids will exhibit fluctuations of the magnitude of the MR effects which will be intolerable from a technological point of view. This problem can only be overcome by a controlled positioning of the MnAs within the sample. Apparently such a degree of control implies the possibility of actively tailoring the MR effects of such devices. Figure 3 emphasizes this aspect by presenting calculations for designed cluster arrangements, labeled (a)-(d). Arrangement (a), where the clusters form two lines perpendicular to the current direction, shows the largest MR effects, about a factor of 4 larger than for typical random arrangements of clusters of the same $x_{C}=22 \%$ (see Fig. 1). In contrast, arrangement (d), where the clusters form two lines parallel to the current direction, exhibits MR effects similar to the pure matrix material in Fig. 1, as the main current path passes through the matrix and is not affected by clusters. Arrangement (c) with an almost homogeneous distribution of clusters shows a MR behavior which is in magnitude very similar to that of random arrangements. The observed scaling of the positive MR effects solely arises from the cluster arrangement as all other parameters have been kept constant in the calculation. Therefore, it is a pure geometric effect which is caused by effective current blocking by the clusters in an applied magnetic field due to spin conservation and a corresponding extension of the current path. This is further underlined by the MR curve of arrangement (b) where the two cluster lines are no longer covering the entire width of the sample. The main current path with an applied field is meandering around the two cluster lines whereas in zero field it passes through the clusters. This extension of the current path by applying a magnetic field leads to a positive MR effect still larger than for the random arrangements.

In summary, controlling the spatial arrangement of MnAs clusters within GaAs:Mn/MnAs hybrids allows one to actively tune the MR effects of these composite materials. Novel developments in the growth of granular hybrid systems, which yield a control of the individual cluster positions, in conjunction with theoretical modeling on a predictive level can be used to systematically optimize the properties of such systems for technological applications in miniaturized devices. Thus, the problem of statistical fluctuations of the desired properties due to the spatial randomness of the cluster arrangement can be overcome offering new possibilities of employing granular systems in devices.

We are grateful for financial support by the DFG in particular by the European Graduate College "Electron-electron interactions in solids."

${ }^{1}$ I. S. Beloborodov, A. V. Lopatin, V. M. Vinokur, and K. B. Efetov, Rev. Mod. Phys. 79, 469 (2007).

${ }^{2}$ A. Bajpai and A. K. Nigam, J. Appl. Phys. 101, 103911 (2007).

${ }^{3}$ P. N. Hai, M. Yokoyama, S. Ohaya, and M. Tanaka, Physica E (Amsterdam) 32, 416 (2006).

${ }^{4}$ C. Binns, K. N. Trohidou, J. Bansmann, S. H. Baker, J. A. Backmann, J.-P. Bucher, D. Kechrakos, A. Kleibert, S. Louch, H.-K. Meiwes-Broer, G. M. Pastor, A. Perez, and Y. Xie, J. Phys. D 38, R357 (2005).

${ }^{5}$ S. Hara and A. Kuramata, Nanotechnology 16, 957 (2005).

${ }^{6}$ L. Li, A. M. Crawford, S. X. Wang, A. F. Marshall, M. Mao, T. Schneider, and R. Bubber, J. Appl. Phys. 97, 10 F907 (2005).

${ }^{7}$ H. A. Krug von Nidda, T. Kurz, A. Loidl, T. Hartmann, P. J. Klar, W. Heimbrodt, M. Lampalzer, K. Volz, and W. Stolz, J. Phys.: Condens. Matter 18, 6071 (2006)

${ }^{8}$ C. Brosseau, J. B. Youssef, P. Talbot, and A.-M. Kohn, J. Appl. Phys. 93, 9243 (2007).

${ }^{9}$ G. Binasch, P. Grünberg, F. Saurenbach, and W. Zinn, Phys. Rev. B 39, 4828 (1989).

${ }^{10} \mathrm{G}$. Moore, Electronics 38, 114 (1965).

${ }^{11}$ S. Hara and T. Fukui, Appl. Phys. Lett. 89, 113111 (2006).

${ }^{12}$ J. Noborisaka, J. Motohisa, S. Hara, and T. Fukui, Appl. Phys. Lett. 87, 093109 (2005)

${ }^{13}$ C. Michel, Theoretical Studies of Spin-Dependent Transport Phenomena (Cuvillier, Göttingen, 2007).

${ }^{14}$ C. Michel, S. D. Baranovskii, P. Thomas, W. Heimbrodt, M. T. Elm, P. J. Klar, B. Goldlücke, U. Wurstbauer, M. Reinwald, and W. Wegscheider, J. Appl. Phys. 102, 073712 (2007).

${ }^{15}$ C. Michel, C. H. Thien, S. Ye, P. J. Klar, W. Heimbrodt, S. D. Baranovskii, P. Thomas, M. Lampalzer, K. Volz, W. Stolz, and B. Goldlücke, Superlattices Microstruct. 37, 321 (2005).

${ }^{16}$ P. J. Wellmann, J. M. Garcia, J. L. Feng, and P. M. Petroff, Appl. Phys. Lett. 73, 3291 (1998).

${ }^{17}$ H. Akinaga, J. De Boeck, G. Borghs, S. Miyanishi, A. Asamitsu, W. Van Roy, Y. Tomioka, and L. H. Kuo, Appl. Phys. Lett. 72, 3368 (1998).

${ }^{18}$ S. Ye, P. J. Klar, T. Hartmann, W. Heimbrodt, M. Lampalzer, S. Nau, T. Torunski, W. Stolz, T. Kurz, H. A. Krug von Nidda, and A. Loidl, Appl. Phys. Lett. 83, 3927 (2003).

${ }^{19}$ W. Heimbrodt, P. J. Klar, S. Ye, M. Lampalzer, C. Michel, S. D. Baranovskii, P. Thomas, and W. Stolz, J. Supercond. 18, 315 (2005).

${ }^{20}$ S. A. Solin, T. Thio, D. R. Hines, and J. J. Heremans, Science 289, 1530 (2000).

${ }^{21}$ C. Michel, M. T. Elm, S. D. Baranovskii, P. Thomas, W. Heimbrodt, B. Goldlcke, and P. J. Klar, Prog. Solid State Chem. 5, 819 (2008). 